SUNY-NTG-94-59

Dec. 1994

\title{
Hydrodynamics near the QCD Phase Transition: Looking for the Longest-Lived Fireball
}

\author{
C.M. Hung and E.V. Shuryak \\ Department of Physics \\ State University of New York at Stony Brook \\ Stony Brook, New York 11794
}

\begin{abstract}
We propose a new strategy for the experimental search of the QCD phase transition in heavy ion collisions: One may tune collision energy around the point where the lifetime of the fireball is expected to be longest. We demonstrate that the hydrodynamic evolution of excited nuclear matter does change dramatically as the initial energy density goes through the "softest point" (where the pressure to energy density ratio reaches its minimum). For our choice of equation of state, this corresponds to $\epsilon_{i} \approx 1.5 \mathrm{GeV} / \mathrm{fm}^{3}$ and collision energy $E_{L A B} / A \sim 30 \mathrm{GeV}$ (for $\mathrm{Au}+\mathrm{Au}$ ). Various observables seem to show distinct changes near the softest point.
\end{abstract}


The main objective of experiments related with high energy heavy ion collisions is the production of sufficiently long-lived and well-equilibrated multi-particle systems. This allows a macroscopic description of the system, thus providing information about the Equation of State (EOS). More specific aims are to locate the QCD phase transition and to study a new state of matter, the quark-gluon plasma. One obvious way to do this is to reach very high energies, such as at RHIC and LHC, so that the plasma is produced well above the critical region and may reveal itself by outshinning the backgrounds.

Meanwhile, related experiments are performed at Brookhaven AGS (10-14 $\mathrm{GeV} / \mathrm{A}$ ) and CERN SPS (200 GeV/A), and one of the issues is the magnitude of transverse flow. As noticed long ago [1, 2], near the QCD phase transition the EOS is especially soft, leading to a significant reduction of transverse expansion (as compared to what it would be without phase transition, e.g. for ideal pion gas EOS). Available AGS/SPS data have confirmed this prediction: in particular, no significant growth of the average transverse momentum $\left\langle p_{t}\right\rangle$ with the multiplicity (or transverse energy) is observed. Better $\pi \pi$ interferometry data may help to get quantitative understanding of the transverse expansion issue: current data indicates that at higher (SPS) energies it seems to be even weaker than at lower (AGS) ones. Recent results [3] for non - central collisions have revealed "reaction plane" effects, similar to what was done before at BEVALAC: but it is still premature to conclude whether these data are useful to restrict the EOS.

In this letter we propose to look at the problem from a different angle. The "softness" of the EOS may affect not only the transverse, but the longitudinal expansion as well, leading in principle to a longer lifetime of the excited system. However, this can only happen if the initial conditions are tuned to a narrow range around the softest point. As a result, certain observables should show sharp and specific dependence on the collision energy around this point.

To demonstrate the qualitative consequences of the proposed idea, we start with the simplest description possible, namely the non-viscous baryon- 
free hydrodynamics with Fermi-Landau initial conditions. We have indeed found that the central lifetime of the system increases by a factor 2.5 in a relatively narrow interval of collision energies. Clearly more sophisticated models would modify this number, but we are confident that the main features would persist: The total lifetime should have a maximum near the indicated energy region, with multiple observable consequences.

Turning to theoretical expectations on the EOS, many important issues concerning the QCD phase transition are not yet resolved (e.g. we do not know whether at physical values of the quark masses it is first order transition, or a rapid crossover). However, it is rather well established that dramatic changes take place in a narrow temperature interval $\Delta T \sim 5 \mathrm{MeV}<<$ $T_{c}$, e.g. the energy density changes by about an order of magnitude, from $\epsilon_{\min }$ to $\epsilon_{\max }$. With a somewhat broader use of the terminology, one may refer to this interval as a generalized mixed phase. Although it corresponds to narrow (or zero) intervals in $\mathrm{T}$ and $\mathrm{p}$, the mixed phase dominates the space-time evolution of the system created at AGS/SPS energies.

The particular EOS to be used below corresponds to zero baryon charge density, and it interpolates between the "virial hadron gas" [4] (with the speed of sound $d p / d \epsilon=c_{s}^{2}=0.19$ ) and the quark-gluon plasma (where the bag constant is $B=0.32 \mathrm{GeV} / \mathrm{fm}^{3}$ ). Phase transition (smoothened for numerical purposes) occurs at $T_{c}=160 \mathrm{MeV}$. We show this conventional EOS in an unconventional way in Fig.1, eliminating $\mathrm{T}$ and plotting the (hydrodynamically relevant) ratio $p(\epsilon) / \epsilon$ versus $\epsilon$. It emphasizes the existence of a minimum at $\epsilon=\epsilon_{\max } \approx 1.5 \mathrm{GeV} / \mathrm{fm}^{3}$, the softest point.

Since we consider only central collisions, our calculations are based on the usual equations of ideal relativistic hydrodynamics with axial symmetry $(2+1$ D) The first-order Lax finite difference scheme is used to solve the equations numerically. Energy and entropy conservation is monitored, and we have reproduced results of several earlier works to ensure that technical aspects are under control. The major uncertainties of hydrodynamic calculations are the initial conditions: Keeping them as simple as possible, we assume zero initial velocity and thermalization in a uniform disk with longitudinal 
thickness equal to the Lorentz-contracted nuclear diameter. The total energy of the hydrodynamical subsystem is taken to be half [5] of $\sqrt{S}$. The reactions chosen are central $\mathrm{Au}+\mathrm{Au}$ collisions at varying energies, from $200 \mathrm{GeV} / \mathrm{A}$ (SPS) to $10 \mathrm{GeV} / \mathrm{A}$ (AGS).

As the collision energy is varied, radical changes in space-time evolution are evident: (a) At high energy end, we have violent longitudinal expansion, similar to the well known scale-invariant solution [6]. (b) Close to the "softest point", we found a slow burning regime. In Fig. 2 we show the space-time evolution for these two cases. The two pictures are qualitatively different in almost every aspect. In particular, the contours of longitudinal velocity (the short-dashed ones) in case (a) are a set of straight lines, pointing toward the origin: this implies Hubble-like inertial explosion, without acceleration. Case (b) is completely different: the expansion velocity is zero till the approach of the (slowly propagating) burning front (boundary between mixed and hadronic phase), where acceleration takes place.

Energy dependence of some global parameters of space-time evolution is shown in fig.3 (a). The maximal lifetime of the mixed phase $\tau_{M}$ (measured at $z, r_{t}=0$ ) displays a clear peak, reaching the value $\tau_{M} \approx 25 \mathrm{fm} / \mathrm{c}$. (The particular collision energy at which it happens depends on the specific choice of initial conditions: but the existence of a relatively narrow peak and its height is a rather stable feature.) However, longer lifetime does not automatically imply higher production yields: the price for it is smaller spatial volume. This is demonstrated in the same figure by plotting the total 4 -volume occupied by the mixed phase $V_{M}$ : its energy dependence has only a "shoulder" at the same point.

Still, such radical changes in the space-time evolution should have observable consequences. We have calculated one and two-body spectra of secondary hadrons, produced at the decoupling (or freeze-out) surface: These results will be reported elsewhere, in an expanded version of this paper. We have also evaluated $\gamma$ and $e^{+} e^{-}$production and compared our results with preliminary data [7, 8] from SPS in a separate letter [9], where details of this calculation can be found. We show here only sample results related with 
the energy dependence of observables. In Fig.3(b) we plot the height and the width of predicted rapidity distribution of the $e^{+} e^{-}$pairs (for simplicity, only $M=0.5 \mathrm{GeV}$ is shown, which is representative for other $\mathrm{M}$ values). Both quantities show a noticeable change in energy dependence around the "softest point" of the EOS. The large height and the small width of the rapidity distribution at $E_{L A B} \sim 30 \mathrm{GeV}$ are a direct consequence of the long-lived fireball, radiating at rest. Similar results follow for photon production.

Note that experimental measurements of $\gamma, e^{+} e^{-}$production at $E_{L A B} / A \sim$ $30 \mathrm{GeV}$ can be made by the existing detectors WA80 and CERES, provided SPS runs at these energies. Should the predicted specific behaviour in the dilepton and photon production be detected, it will single out the location of the softest point of the EOS. Many other observables should also be modified in this region. A slow-burning fireball, with lifetime twice longer than usual, and with much smaller size, could be identified by $\pi \pi, K K$ interferometry. Possible modifications of strangeness production or $J / \psi$ suppression in this region are also interesting possibilities.

Summarising, we have suggested a new strategy in the search for the QCD phase transition. The proposal is to repeat measurements with existing detectors, scanning downward from the nominal SPS collision energy. Photon and dilepton spectra are shown to be sensitive to the lifetime, and they can be used to locate the "softest point" of the QCD equation of state.

\section{References}

[1] E.Shuryak and O.V.Zhirov, Phys.Lett.89B (1979) 253-255.

[2] L.van Hove, Z.Phys.C21 (1983) 93; K.Kajantie, M.Kataja, L. McLerran and P.V.Ruuskanen, Phys.Rev.D34 (1986) 2746; S.Chakrabarty, J.Alam,D.K.Srivastava, B.Sinha, Phys.Rev.D46 (1992) 3802.

[3] J.Barette et al (E877 Collaboration), Observation of Anisotropic Even Shapes..., SUNY-RHI-94-8, Subm. to Phys.Rev.Lett.; S.Voloshin and 
Y.Zhang, Flow Study in Relativistic Nuclear Collisions by Fourier Expansion of Asimuthal Particle Distributions, Subm. to Phys.Rev.C

[4] E.Shuryak, Yadernaya Fis. (Sov.J. of Nucl. Phys.) 16 (1972) 395; R. Venugopalan and M. Prakash, Nucl. Phys. A546 (1992) 718.

[5] Another half is attributed to non-thermalized nucleons and their decay products: we do not attempt to describe spectra in the fragmentation regions. We also ignore effect of the excess baryons at central rapidities, which is only about $10 \%$ correction at the SPS end of our energy interval, but larger at the lower end.

[6] J.D.Bjorken, Phys.Rev.D27 (1983) 140.

[7] R.Santo, Nucl.Phys. A566(1994) 61c. Single Photon and Neutral Meson Production from WA80, IKP-MS-93/0701, Munster 1993.

[8] A.Drees, Nucl.Phys. A566(1994) 87c. Th.Ulrich, Low-Mass Electron pair production in p-Be, p-Au and S-Au Collisions at CERN SPS Energies, Heidelberg preprint, Sept.11, 1994.

[9] C.M. Hung and E. Shuryak, The space-time picture of heavy ion collisions and $\gamma, e^{+} e^{-}$production, Stony Brook preprint, SUNY-NTG-94-58 


\section{Figure captions:}

Fig.1 The equation of state used, shown as pressure to energy density ratio $p / \epsilon$ versus $\epsilon$.

Fig.2 Comparison of space-time evolution for central $\mathrm{Au}+\mathrm{Au}$ collisions at (a) $129 \mathrm{GeV} / \mathrm{A}$ and (b) $30.9 \mathrm{GeV} / \mathrm{A}$ (corresponding to the longest fireball lifetime). Solid lines show the contours of fixed energy density at the timelongitudinal coordinate $(\mathrm{t}, \mathrm{z})$ plane, at $r_{t}=0$. (The boundaries between quark $(\mathrm{Q})$, mixed $(\mathrm{M})$ and hadronic $(\mathrm{H})$ phases are shown by the thicker lines, which correspond to energy densities $1.5,0.31$, and $0.13 \mathrm{GeV} / \mathrm{fm}^{3}$ ) The short-dashed lines are contours of fixed longitudinal velocity, starting from $0.1 \mathrm{c}$ for the left-most contour and increasing by steps of $0.1 \mathrm{c}$ towards the right.

Fig.3 (a) Lifetime $\tau_{M}$ of the mixed phase (dashed line, right scale) and the corresponding space-time volume $V_{M}$ (solid line, left scale) (b) The height of the dilepton rapidity distribution $d N_{e+e-} / d y(M=0.5 \mathrm{GeV}, y=0)$ (dashed line, right scale) and its full width at half maximum (solid line, left scale) versus the collision energy. 
This figure "fig1-1.png" is available in "png" format from: http://arxiv.org/ps/hep-ph/9412360v1 


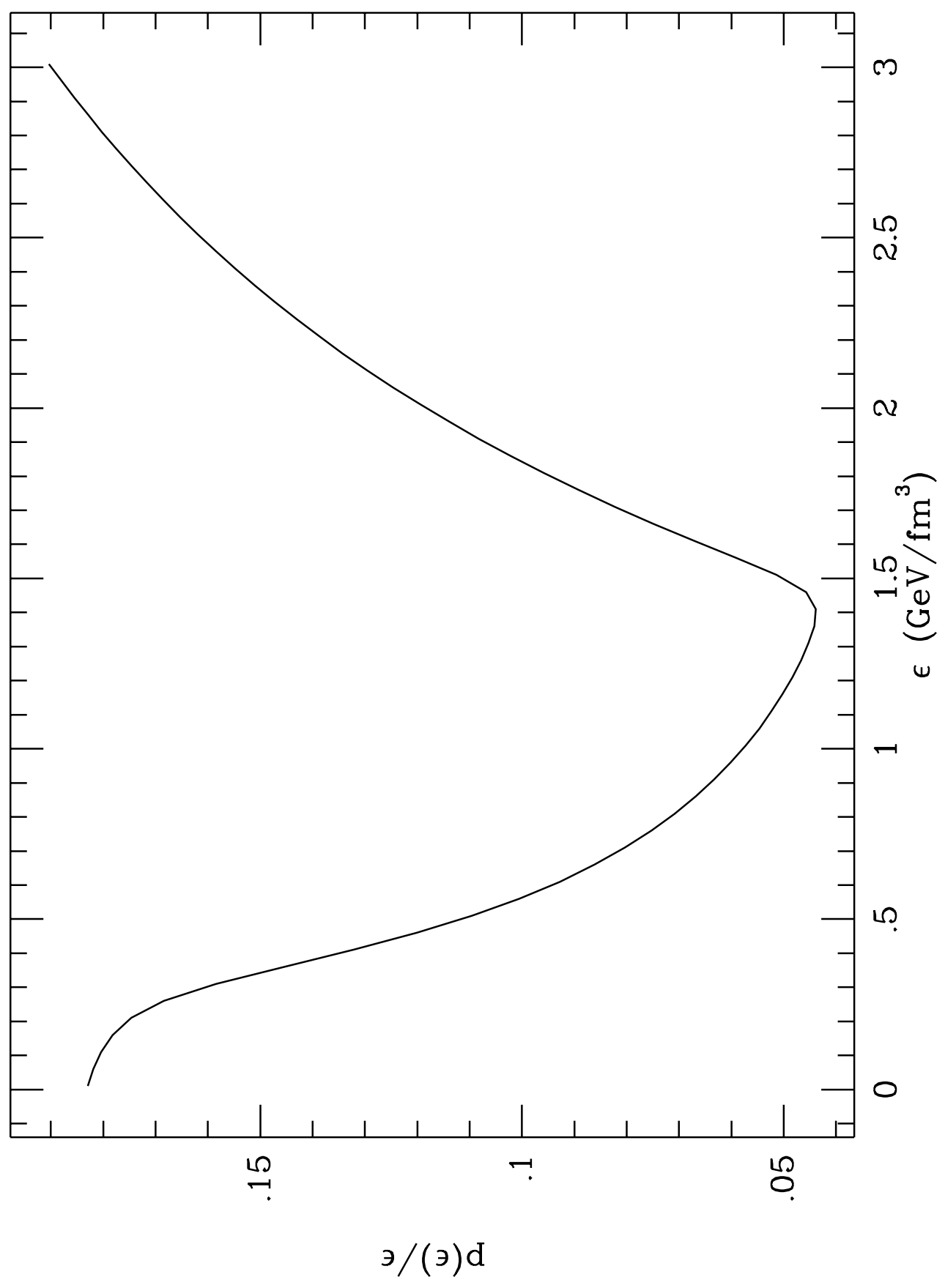


This figure "fig1-2.png" is available in "png" format from: http://arxiv.org/ps/hep-ph/9412360v1 

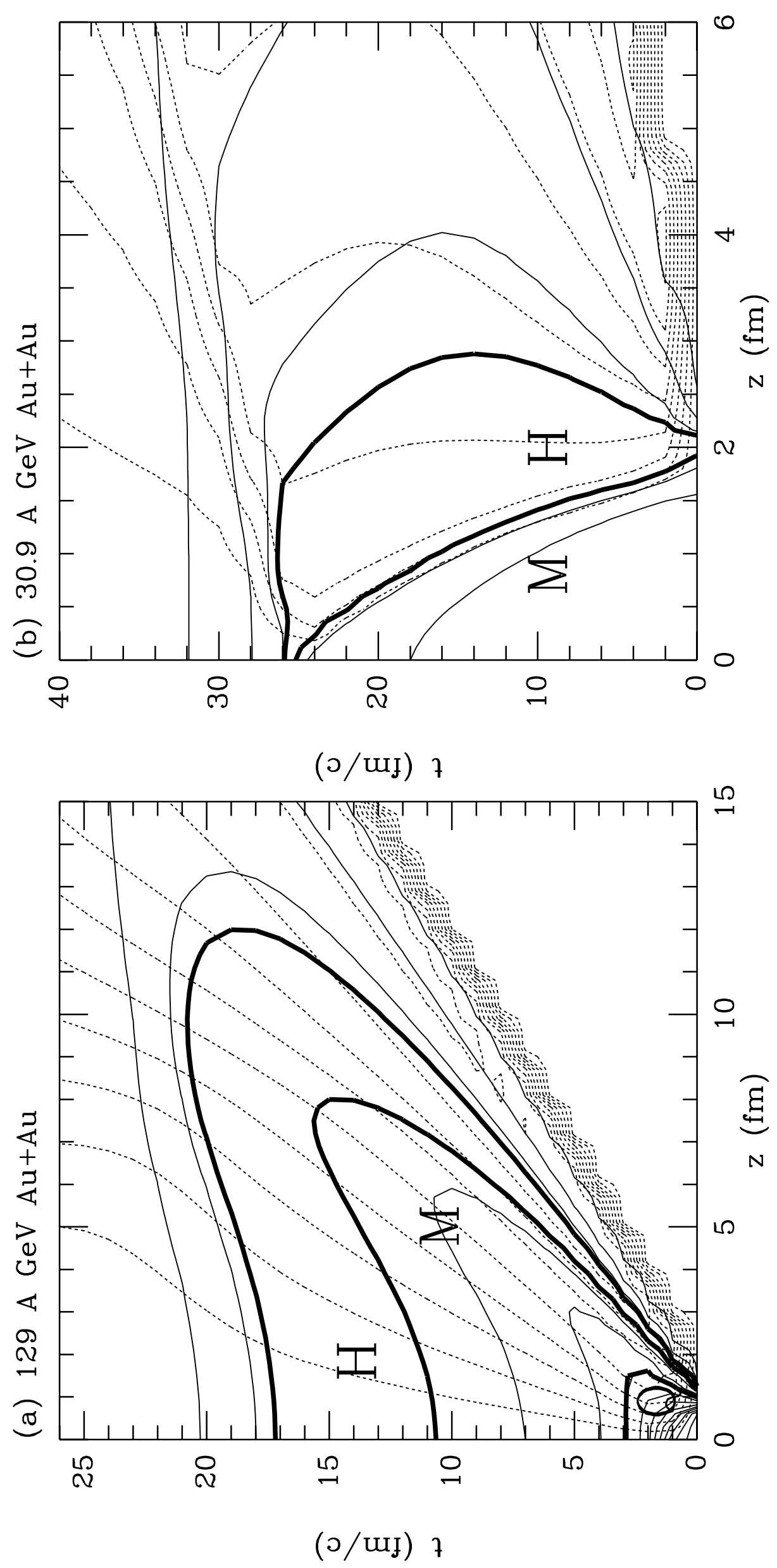
This figure "fig1-3.png" is available in "png" format from: http://arxiv.org/ps/hep-ph/9412360v1 


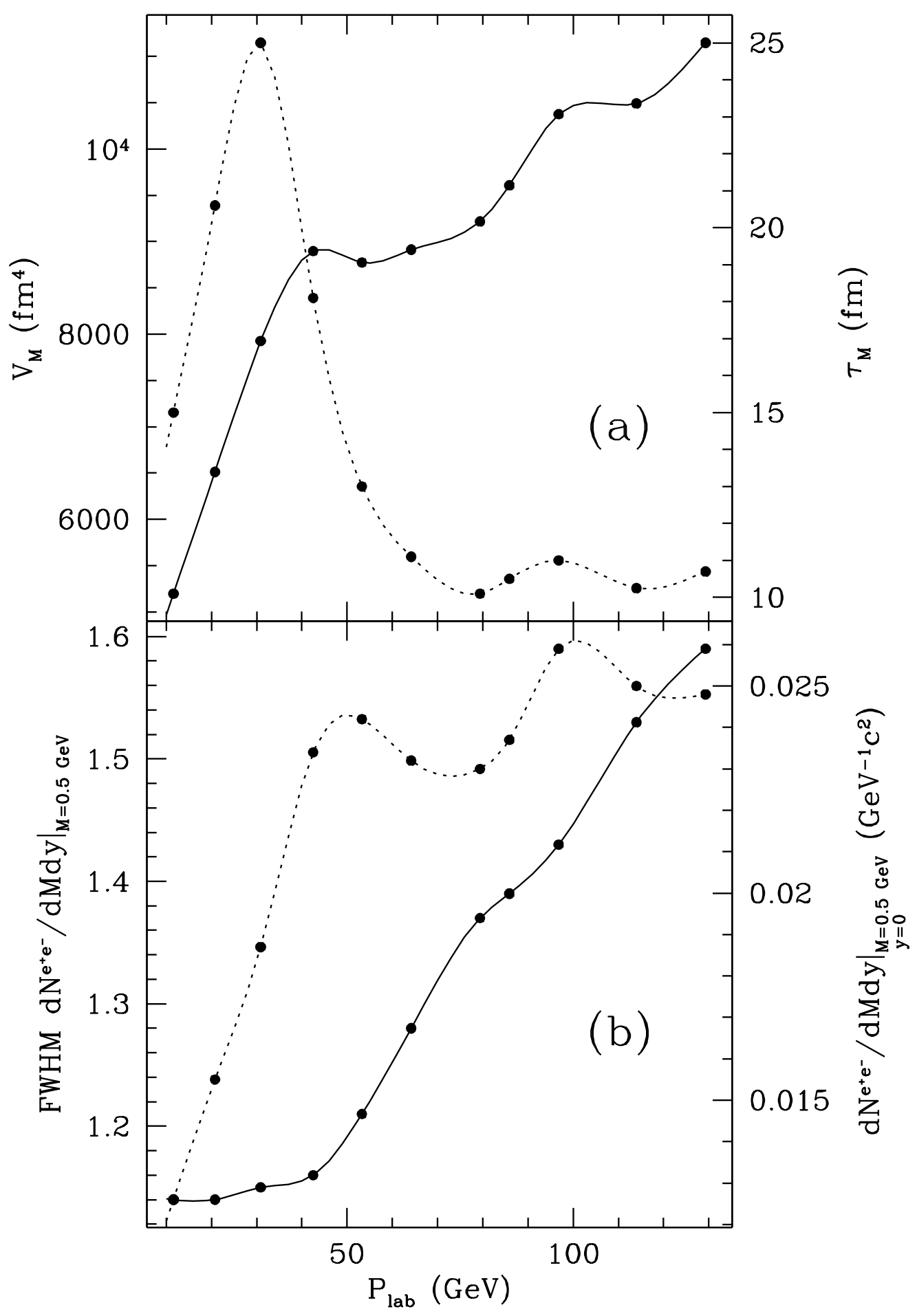

